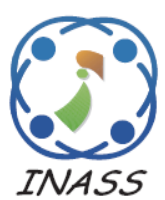

\title{
HM2LP: Hybrid Multilevel Multihop LEACH Protocol for Conserving Energy in Large Area WSN
}

\author{
Monika Rajput ${ }^{1 *}$ \\ Sanjay Kumar Sharma ${ }^{1}$ \\ Pallavi Khatri $^{2}$ \\ ${ }^{I}$ Department of Computer Science, Banasthali Vidyapith, Rajasthan, India \\ ${ }^{2}$ Department of Computer Science and Engineering, ITM University, M.P., India \\ * Corresponding author's Email: monika.rajput03@yahoo.com
}

\begin{abstract}
Wireless Sensor Network (WSN) for a large area is the need of the hour due to ever-growing demand on the fly services in different application fields. More senders deployed in a large area will generate an enormous amount of data and will communicate to the base station (BS). The distance of a device from the BS will affect the energy of the transmitting sensor. While collecting data at the BS required distance of the route to travel by the data packets is also longer. More energy of the nodes is consumed during transmission, reception, and data aggregation phases. Systems designed for small networks perform poorly in large networks due to more energy dissipation. This work proposes using Rendezvous nodes (RN) to reduce energy consumption. The network is divided into grids. Each grid has an RN. All the selected cluster head $(\mathrm{CH})$ sends their data to the RN of its zone, and then the RN transmits this data to the BS. Using this method, the traffic load of the network is reduced. The lifetime of the network is improved, which is the fundamental need of large WSNs. Existing protocols Energy Efficient Sleep Awake Aware (EESAA), Energy Efficient Scalable Routing Algorithm (EESRA), Modified Invasive Weed Optimization Clustering Approach (M-IWOCA), and Low Energy Adaptive Clustering Hierarchy (LEACH) are also implemented in the proposed scenario. Total network lifetime shows growth by $12 \%$ using the developed Hybrid Multilevel Multihop LEACH Protocol (HM2LP).
\end{abstract}

Keywords: WSN, Lifetime, Clustering, Rendezvous nodes, Energy efficiency, Large network.

\section{Introduction}

Wireless Sensor Network is configured to operate on battery-operated supply and generates data by sensing the field information. The networks are capable of generating data at a high rate for the occurrence of an event. Deployment of a large area network requires topological arrangements to reduce the power consumption required for transmission and reception of the data. The efficiency in communication with respective zonal arrangements can reduce the significant amount of energy and hence in order the total lifespan of the wireless sensor network. The total energy available from battery power supplies is required to be utilized efficiently that there will be less count of deaths of the nodes with respect to time. The clustering approach shows better performance over non clustered networks in which LEACH is a basic cluster-based protocol in the WSN domain. The $\mathrm{CH}$ selection, its location from the BS, and other sensor nodes is the main aspect in which the death of $\mathrm{CH}$ is responsible to count as the death of node in WSN. To save such occurrences of deaths in large area networks where coverage of WSN is very large, the selection of $\mathrm{CH}$ requires optimized processing, and selection of the right node as $\mathrm{CH}$ with respect to its residual energy is a fundamental approach considered and presented in this paper.

This work focuses on the selection of nodes as $\mathrm{CH}$, handing over the charge of the $\mathrm{CH}$ to some other node, and continuously monitoring the energy level of $\mathrm{CHs}$ for $\mathrm{CH}$ selection attempts. The $\mathrm{CH}$ is also responsible for handover the data towards the BS. The cases may arise when BS is not in the direct range of $\mathrm{CH}$ and hence other $\mathrm{CHs}$ who are in direct 
contact with BS can do the job of routing to collect the data from a particular $\mathrm{CH}$. The main ideology of the paper is dividing the large WSN into small zones with the deployment of $\mathrm{RN}$ as virtual BSs and selecting $\mathrm{CHs}$ with the use of an adaptive neurofuzzy inference system with optimization using the firefly algorithm. The ANFIS selects $\mathrm{CH}$ using residual energy, traffic load, and distance parameters for selecting $\mathrm{CH}$. The attempt of selection of $\mathrm{CH}$ directly depends on the expiry of the timer. The value of the timer depends on the energy consumption rate. The energy consumption rate is dependent on the traffic load on a particular $\mathrm{CH}$. Thus, the synchronization, $\mathrm{CH}$ selection attempts, and collection of data for maximum network life is the core approach considered in the development of a protocol for large area networks, presented in this paper. The primary contribution of this article is the updated clustering approach, optimal $\mathrm{CH}$ selection, complexity reduction, and wide area WSN combined with zonal RN for overall network energy efficiency enhancement to improve the WSN lifespan.

This research paper is organized as follows: Section 1 is Introduction. Literature surveys of the existing methods are discussed in section 2. Details of the proposed work and methodology are presented in Section 3. Section 4 contains the experimental setup. Section 5 shows the results and discusses and finally concluding comments in section 6 .

\section{Literature survey}

There are several approaches available that promise to improve energy efficiency in WSN. This section focuses on approaches that make use of the LEACH protocol as a platform protocol for the sake of progress and improvement in energy efficiency.

Several types of research are going on in the arena of WSN. The key objective is to maximize the lifespan of the network by some means declining the consumption of vitality inside the network. A decent way to increase the lifespan of the network is by using multi-hopping. In this method each node sends its data to other nodes and after that data is transfer to the sink. By doing so total distance is decreases and the effect of total energy consumption is also decreases. However, the drawback of using this method would be that node closer to the BS would die early. Clustering is also used to reduce the energy consumption in the network. In this approach, the network is divided into clusters. Each cluster has a $\mathrm{CH}$ which is responsible for the communication. All the non- $\mathrm{CH}$ nodes send the data to the $\mathrm{CH}, \mathrm{CH}$ aggregates the data and transmit it to the BS. The leading problem of the clustering technique is that $\mathrm{CH}$ is consumed more energy as compared to the other nodes and due to that $\mathrm{CH}$ dies early.

LEACH [1] is an illustrative clarification based on probability gathering. LEACH is a grouping procedure where $\mathrm{CH}$ selects sporadically. It has two phases 1) Setup Phase and 2) Steady Phase. In the first phase selection of $\mathrm{CH}$ is through probability rate and in the second phase files are transferred to the BS. When the clusters are shaped, $\mathrm{CH}$ starts receiving the data from nodes. Every node transfers data to the $\mathrm{CH}$ according to the TDMA in LEACH. Energy consumption of the LEACH is less as compared to the other conservative protocols and has a better network lifespan.

To examine the energy consumption of $\mathrm{CH}$ and non- $\mathrm{CH}$ nodes, the distance-based $\mathrm{CH}$ selection algorithm LEACH-DT is proposed in [2], which considers the distance between sensor nodes and BS to enhance the energy depletion in the network. The proposed method overtakes as compare to the existing method in terms of the node lifetime and the first node died time. Reproductions show that the LEACH-DT is better than the basic LEACH protocol with a $10 \%$ improved network lifetime.

Modern energy efficient computational techniques for improving $\mathrm{CH}$ selection have been presented in [3]. However, the complexity of the algorithm and the network connectivity issues are still not considered. This work proposes a novel multi-objective algorithm known as Multi-objective imperialist competitive algorithm (MOICA) for optimizing $\mathrm{CH}$ selection. The objective of this work was to analyze the performance of MOICA for optimizing routing and $\mathrm{CH}$ selection. The results indicated that it can achieve a lifetime performance improvement of over $56.32 \%$ as compared to the basic LEACH protocol.

A new energy-efficient routing protocol for Wireless Sensor Networks (WSNs) is proposed in [4]. The goal of this work is to develop an energyefficient routing protocol for WSNs. It uses the Yellow Goatfish algorithm to determine the optimal $\mathrm{CHs}$ for each BS and the sensor nodes. Experiments show that this method can reduce the energy consumption and improve the stability of the network as compared to the Distributed EnergyEfficient Clustering Algorithm (DEEC), LEACH, and Stable Election Protocol (SEP).

The author suggested Sampling-Based Spider Monkey Optimization for Energy-Efficient ClusterHead Selection (SSMOECHS) in WSNs in [5]. In SSMOECHS, a $\mathrm{CH}$ selection method employs sampling to account for real placement of nodes. 
The best $\mathrm{CHs}$ are found by sampling and then optimized utilizing a modified sample-based spider monkey optimization SMO technique, which reduces the divergence between the ideal and real $\mathrm{CH}$ node positions while increasing energy efficiency. In both homogeneous and heterogeneous configurations, the findings are compared to comparable protocols such as low-energy adaptive clustering hierarchy centralized (LEACH-C), particle swarm optimization clustering protocol (PSO-C), and threshold-sensitive energy-efficient delay-aware routing protocol based on SMO (SMOTECP). According to the research, the SSMOECHS increases the longevity and stability of WSNs.

In [6], a novel energy-saving algorithm is developed, and its efficiency is compared to that of the LEACH and Hybrid Energy-Efficient Distributed clustering (HEED) algorithms. In this work, weighting coefficients are used to offer a new technique of clustering and selecting $\mathrm{CHs}$ based on the energy efficiency and residual energy of each node. The performance of the entire network has been enhanced using the suggested technique, which focuses on the residual energy of each node and the likelihood of becoming $\mathrm{CH}$. It has been proven that this work has a significant impact on energy savings and network lifespan.

The energy constraint of sensor nodes makes the network lifespan of WSNs a significant challenge. This problem is exacerbated in large-scale sensor networks, where huge amounts of energy are used for big data groups and packet transfers. For solving this problem an energy efficient clustering method called Distributed Entropy Energy-Efficient Clustering Algorithm (DEEEC) is proposed in [7] for Heterogeneous Wireless Sensor Networks (HWSNs). There are two major phases to the DEEEC Algorithm. In the first phase, temporary $\mathrm{CHs}$ are identified. In the second stage, if any cluster member has higher residual energy, the temporary $\mathrm{CHs}$ will be modified. Nodes with high energy have a chance to become the $\mathrm{CHs}$ decided by the above two steps, which are designed for $\mathrm{CH}$ selection. When compared to the existing standard clustering procedures utilized in heterogeneous WSNs, the simulated results of the proposed DEEEC Algorithm show good outcomes in terms of energy and enhanced longevity.

The author proposed a new clustering technique for wireless sensor networks called energy-efficient weighted clustering method (EEWC) in [8]. The proposed method used a newly designed fitness weighted fitness function that takes into account clustering quality variables including compactness, separation, and $\mathrm{CH}$ count. The recommended fitness function is used in the evolutionary process to determine the optimal collection of $\mathrm{CHs}$ during the steady-state phase of the LEACH method. The simulation findings show that the EEWC technique outperforms compared to the other clustering approaches such as stable election protocol (SEP), intelligent hierarchical clustering and routing protocol (IHCR), and evolutionary routing protocol (ERP) in terms of stability period, network longevity, and total residual energy.

In [9], the researcher deliberated the difficulty of using numerous RPs to improve the lifespan of WSN. A heuristic and optimal procedure are planned here to report this problem. According to the author optimum procedure is difficult and it's appropriate only for small WSN. The reproduction outcomes show that the heuristic algorithm is ideal using various RP sets that can suggestively extend network lifespan.

Algorithm called LEACH Mobile Sink RN with an altered $\mathrm{CH}$ Selection process (LMRNACH), as well as some modifications to the $\mathrm{CH}$ selection process has been proposed in [10]. The goal of this research was to extend the Network's lifespan. LMRNACH was achieved by changing the $\mathrm{CH}$ selection process, which was influenced by four factors: node distance from the sink, distance between nodes and other $\mathrm{CH}$, leftover energy, and the number of times a node has been $\mathrm{CH}$. In simulations, the proposed method clearly outperformed the traditional LEACH mobile sink and RN node, especially in large network dimensions.

In [11] author has proposed an optimal path selection method to increase the lifespan of the network. A weight is assigned to each sensor node to its hop distance. This method is called Weighted Rendezvous Planning. Set of RPs are selected in such a way as a result the energy depletion for the communication can be compact. All the sensor nodes will send the sensed data to the allocated RP, if some sensor nodes do not have RP, it will send the data to the nearest RP using multi hopping. Results analysis shows that the proposed method consumed less energy and increases the network lifetime.

In [12] rendezvous-based direction-finding procedure is given, which generates a rendezvous area in the center of the system and builds a tree inside that area. Two modes of transmission are given. First, the tree is the direction of the BS and the basis node conveys the information to the BS through this tree. However in the second mode, BS conveys the location to the tree, and the foundation 
node acquires the BS's location from the tree and conveys the information straight to the BS.

The energy consumption of the sensors in the network is a hot topic right now, which is why many researchers are concentrating on it. Author proposed a method called Energy Efficient Sleep Awake Aware (EESAA) [13] to enhance service quality by improving clustering selection strategy to make the network more energy efficient. In this method nodes used sleep and awake technique to save the energy. When nodes have nothing to communicate they go to sleep mode and when have a signal to communicate they will awake and start communicating. The author has covered load balancing protocols including LEACH, EESAA, and Stable Election Protocol (SEP) in this paper, which will help to optimize the network's lifetime, management efficiency, and energy usage. Comparative analysis has been done with LEACH and SEEP. Simulation results show that the EESAA perform better in term of network lifespan and $\mathrm{CH}$ selection process as compared to other two protocols.

The Energy Efficient Scalable Routing Algorithm (EESRA), based on the LEACH protocol, is a novel three-layer WSN routing algorithm introduced in [14] by the author. EESRA was created with the objective of extending the network's lifetime as the network grows in size. In each cycle, a hybrid MAC protocol was employed, which included data sensing sleep and collision avoidance techniques, as well as TDMA slots for data forwarding. Congregations (CGs) were also used to receive sensed data from $\mathrm{CMs}$ in order to better balance load. EESRA is compared to various WSN routing protocols in terms of network performance as network size varies. On large-scale WSNs, simulation findings demonstrate that EESRA is more energy efficient and better at load balancing than other protocols

Richa [15] proposed an energy-efficient MIWOCA protocol. For $\mathrm{CH}$ selection, an optimized genetic algorithm (GA) is used. The fuzzy modeling is the part of GA in which the node with maximum residual energy is selected as $\mathrm{CH}$. In the initialization phase, the network is first divided into clusters using a k-means clustering algorithm. The GA plays a role of selecting new $\mathrm{CH}$ after every round with consideration of residual energy. The $\mathrm{CH}$ is not changed if the residual energy of the existing $\mathrm{CH}$ is sufficient and above the expected threshold. The optimum $\mathrm{CH}$ selection makes use of distance from the center point of cluster and location of nodes participating in the selection process. Simulation results show better performance over LEACH.
The left over energy and density inside the sensor nodes zone radius, the number of dead nodes and the position and velocity of the mobile sink may all influence the energy balance of WSNs. This study [16] presented Cluster-Based Energy Optimization with Mobile Sink (CEOMS), a novel cluster-based energy optimization technique for selecting $\mathrm{CHs}$ by taking into account the influence of the above parameters on the energy balance of WSNs. The proposed technique first constructed the energy density function by analyzing the residual energy rate and density within the neighborhood radius of nodes, which reduced the unpredictability of $\mathrm{CH}$ selection. The energy density function, motion performance function, and adaptive adjustment function all worked together to improve $\mathrm{CH}$ self-adaptability, balance network load, reduce data latency, and extend network life. Furthermore, when the monitoring region is vast, the suggested method only employs a mobile sink, resulting in fractional data loss and delay.

The important finding from the selective literature survey is that clustering plays an important role in energy efficiency enhancement. The $\mathrm{CH}$ charge periodic handover is necessary for conserving the energy of all the nodes and maximal use for sensor data collection. The existing protocols show that the strategy for $\mathrm{CH}$ selection makes requires consideration of residual energy and distance. The periodic handover of $\mathrm{CH}$ charge to other nodes requires fuzzy rules consideration for right $\mathrm{CH}$ selection. The $\mathrm{CH}$ selection attempts are also networked overhead increasing aspects that increase energy consumption. Another important consideration is that the $\mathrm{CH}$ to $\mathrm{BS}$ communication also involves other nodes in the route which increases unnecessary consumption and overall lifetime decrement.

To make the network more energy efficient, this research proposes the HM2LP. The suggested protocol is being developed to solve the abovementioned shortcomings in the present protocol and the use of an $\mathrm{RN}$ node may support more energy efficiency by acting as a virtual BS.

\section{Proposed methodology}

The suggested study focuses on clustering, reducing the number of clustering attempts, selecting $\mathrm{CH}$ based on distance and energy in combination with ANFIS rules, and using RN for energy efficiency. The core concern of the WSN is the depletion of energy. The objective of this research work is the development of the protocol for large area WSN to maximize energy efficiency and 


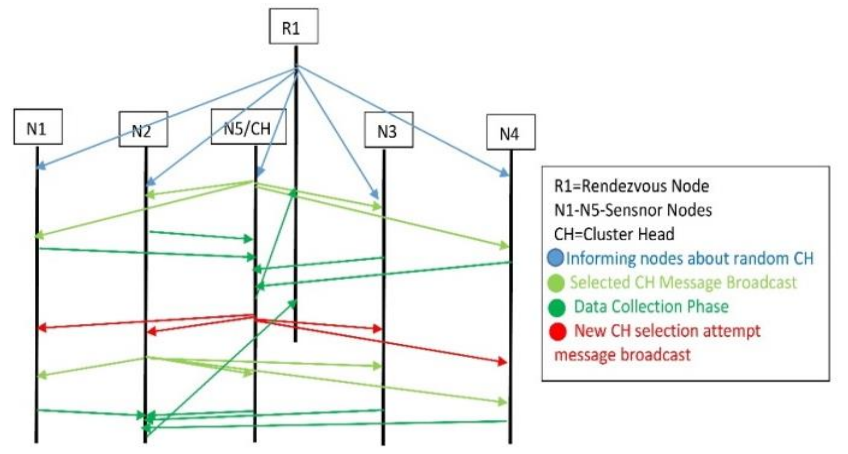

Figure. 1 HM2LP protocol working flow

maximize the lifespan of the network. The HM2LP with $\mathrm{RN}$ is proposed here for large area WSN to maximize the energy of the network. The clusteringbased protocol mechanism is considered a fundamental approach in the development of the proposed protocol. RN are virtual BS nodes considered with high battery capacities and can communicate directly with BSs.

The steps of WSN configuration and details of the protocol are:

1. Deploy random sensor nodes in a large area network.

2. Deploy RNs in a grid manner in the same network which acts as a virtual BS for a specific zone.

3. The task of RNs is to hand over the data towards the BS collected from $\mathrm{CHs}$ from a specific zone.

4. Initialize the nodes in the network with full battery capacity.

5. Start K-medoid clustering for randomly selecting CHs.

6. Start a collection of data and sending towards the BS via Rendezvous nodes.

7. Estimate the time for a new $\mathrm{CH}$ selection attempt and start the timer.

8. Reselect the $\mathrm{CH}$ when the timer expired.

9. Use new $\mathrm{CH}$ and inform the nodes within the cluster about the new $\mathrm{CH}$.

The HM2LP protocol mechanism consists of two phases in which the initialization phase starts with the decision from RN. Once initialization is complete, the RUN phase starts in which the network continues with continuous stages of tasks depending on conditions set and reset during the run phase. Fig. 1 shows the working of the HM2LP protocol.

\subsection{Initial K-medoid clustering and member association}

In clustering-based methods, the initialization phase is also called as setup phase. The K-medoid
[17] clustering is vectored clustering method in which the first group of $\mathrm{CHs}$ is selected randomly. The distance parameter is used to select the $\mathrm{CH}$ and its member's association. Association of all the sensor nodes proceeds when respective control packets signaling takes place. The K-medoid clustering process starts as soon as $\mathrm{RN}$ sends the $\mathrm{RNCH}$ packet to all the sensor nodes in the zone to inform that the $\mathrm{CH}$ selection process is going to start. After that $\mathrm{CH}$ is selected using the K-Mediod process, and then the selected $\mathrm{CH}$ broadcast the CHSelc packet to all the nodes in the vicinity to start the association process. Nodes that get CHSelc packet send the CHAsso packet towards $\mathrm{CH}$ in vicinity associate with $\mathrm{CH}$.

The association of sensor nodes with $\mathrm{CH}$ depends on the sensor node's choice. The CHSelc from nearest $\mathrm{CH}$ reaches earlier than other $\mathrm{CHs}$ and hence first come first association method is used. Once the CHAsso message is sent by sensor nodes to $\mathrm{CH}$, the $\mathrm{CH}$ acknowledges with CONFIRM packet.

\subsection{RUN phase}

In the RUN phase, there are $\mathrm{CHs}$ by now available from the initialization phase, which is responsible to collect the data from all the sensor nodes, aggregate it and send it to the RN. RN collects all the data and sends it to the BS.

During this phase after communication, the energy level of $\mathrm{CH}$ decreases earlier than other sensor nodes in that cluster due to additional packet data aggregation tasks. The rate of this energy consumption is estimated with respect to the traffic load. The traffic load is measured in terms of packets per second. The energy required for each packet reception and transmission is multiplied by packets per second to get the rate of energy consumption. The formula for this is given in Eq. (1),

$$
E_{a}=E_{p} \frac{P}{t}
$$

where,

$E_{a}=$ Rate of energy consumption per second

$E_{p}=$ energy consumed for each packet

$P$ is the total number of packets in time window t.

A timer is applied to every $\mathrm{CH}$. The $E_{a}$ can be used to estimate the timer. After expiring the timer the $\mathrm{CH}$ selection process starts again to decide that whether to continue with the same $\mathrm{CH}$ or hand over the charge of existing $\mathrm{CH}$ to some other nodes within the cluster.

The timer estimation thus can be given in Eq. (2), 


$$
T=\frac{E_{c}-E_{t h}}{E_{a}}
$$

Where,

$E_{c=}$ Current residual energy of existing $\mathrm{CH}$

$E_{t h}=$ Energy threshold level above which particular sensor node is allowed to become $\mathrm{CH}$

$E_{a}=$ Rate of energy consumption per second

The main advantage of using the timer as a constraint for $\mathrm{CH}$ selection attempt is that the verification of the residual energy above the threshold may not be necessary as, total energy consumed within a particular time period is possible to estimate with respect to the rate of energy consumption which depends on traffic load.

\section{3 $\mathrm{CH}$ reselection}

On the expiry of the timer, the $\mathrm{CH}$ reselection process starts in which ANFIS optimized with firefly algorithm is used to select new CH. ANFIS with firefly is used to optimize the neural network in ANFIS the same way as described in [17]. ANFIS model and FIS parameters for reselection of $\mathrm{CH}$ are defined in [17]. $\mathrm{CH}$ reselection process will start in only those particular clusters in which the timer of $\mathrm{CH}$ is expired.

ANFIS model optimization is possible by optimizing the number of neurons in the ANFIS network [18]. The whole process of the firefly algorithm is illuminated in [19]. The root means square error (RMSE) is used to improve the performance. Every firefly contains an abundant agreement of forerunner and subsequent factors.

The estimated vector of data aspects $(z 1, z 2$, and $z 3)$ and a yield variable (y) and using three fleecy groups through Gaussian-type asset limits given in Eq. (3).

$$
y s=a s z 1+b s z 2+c s z 3+d s z 4+e
$$

Where, $a s, b s, c s, d s$, and es parameters are used for biasing the elements in the ANFIS model. $y s$ is the yield function and $z 1, z 2, z 3, z 4$ are the Data factors.

\section{Proposed algorithm}

Algorithm 1 demonstrates how the proposed technique works. The initialization step begins with the RN transmitting the $\mathrm{RNCH}$ packet to all sensor nodes inside the zone. The RNCH packet tells nodes that the $\mathrm{CH}$ selection procedure is about to begin. The K-mediod method is then used to choose $\mathrm{CH}$. The selected $\mathrm{CH}$ transmits the $\mathrm{CHSelc}$ packet to
Algorithm 1: Hybrid Multilevel Multihop LEACH Protocol (HM2LP)

1. Initialize the network.

2. RN sends the $\mathrm{RNCH}$ to all the sensor nodes within the zone.

3. $\mathrm{CH}$ selection process starts using K-medoid

4. Selected $\mathrm{CHs}$ broadcast the CHSelc to all the nodes.

5. The node association process starts by sending the CHAsso to $\mathrm{CH}$.

6. $\mathrm{CH}$ acknowledges with CONFIRM to the nodes.

7. Evaluate timer value using Eq. (2) and set the timer for the next $\mathrm{CH}$ selection attempt.

8. Selected CHs send the data to the RN.

9. RN sends the collected data to BS

10. for each $\mathrm{CH}$ in the network do

11. check the timer

12. if the timer terminated then

13. Start reselection of the $\mathrm{CH}$ by ANFIS

14. Go to step 4

15. else if the timer is not terminated then

16. Go to step 8

17. end if

18. end loop

all nodes in the region to begin the association process. When a node gets a CHSelc packet, it sends a $\mathrm{CHAsso}$ packet to the nearby $\mathrm{CH}$. The $\mathrm{CH}$ sends a CONFIRM packet in response. Now enter the Run phase, where the timer value is computed using equation 2 and set the timer for each $\mathrm{CH}$. Selected $\mathrm{CH}$ begins sensing data and transmits it to the RN. The collected data is then sent to the BS via the RN. During the RUN phase, the timer is checked to see if it has expired for the particular $\mathrm{CH}$. If it has, the $\mathrm{CH}$ is reselected using ANFIS parameters that employ distance and residual energy as metrics.

\section{Simulation setup}

The implementation of the HM2LP protocol is done in MATLAB for a large area network of 1000 sq. m. Total 1000 nodes are deployed in the network. As per the requirement, a total of $9 \mathrm{RN}$ nodes are deployed in a grid pattern as shown in Fig. 2. The simulation parameters are shown in Table 1 . The existing protocols are also implementing in the proposed scenario.

\section{Results and discussion}

To calculate the performance of HM2LP, it is compared with the EESAA, EESRA, M-IWOCA, LEACH protocols. The key focus of the work is to improve the energy efficiency of the network. 
Performance of the HM2LP is calculated by estimating the number of alive nodes per round, the number of dead nodes per round, estimate the duration from the start of the network process to when the first node died (FND), time taken to reach the last node died (LND), the average residual energy and the average consumed energy. The lifetime of the network is also calculated.

\subsection{Alive and dead nodes per round}

After the successful completion of each round number of alive and dead nodes is being calculated. The $\mathrm{CH}$ selection attempts reduction and changing the charge of $\mathrm{CH}$ before run out are the main reasons that keep the maximum number of nodes alive with respect to iterations. Fig. 3 and 4 gives the study of dead nodes and alive nodes. It has been found that the HM2LP performs better as compared to the EESAA [13], EESRA [14], M-IWOCA [15], and LEACH [1] protocols. A total 6\% improvement is observed in the performance of HM2LP in terms of alive and dead nodes as compared to the other four existing protocols.

\subsection{Residual and consumed energy per round}

Residual energy is the current value of energy in a node after receiving and transmitting packets and consumed energy is the value of energy that is consumed in the data transmission. In other protocols, a residual energy-based threshold is taken into account throughout $\mathrm{CH}$ selection attempts, and

Table 1. Simulation parameters

\begin{tabular}{|c|c|}
\hline Parameter & Value \\
\hline Size of the network & $1000 \times 1000 \mathrm{~m}^{2}$ \\
\hline Total nodes & 1000 \\
\hline Rendezvous nodes & 9 \\
\hline emp & $100 \mathrm{nj}$ \\
\hline Sink location & Center \\
\hline $\begin{array}{c}\text { Initial Energy of each } \\
\text { node }\end{array}$ & $10 \mathrm{~J}$ \\
\hline Transmission energy & $50 \mathrm{uJ}$ \\
\hline Reception energy & $50 \mathrm{uJ}$ \\
\hline Data aggregation energy & $5 \mathrm{uJ}$ \\
\hline Packet size & 4000 \\
\hline Protocols & $\begin{array}{c}\text { HM2LP, EESAA, EESRA, } \\
\text { M-IWOCA, LEACH }\end{array}$ \\
\hline Performance Parameters & $\begin{array}{c}\text { Alive nodes, Dead nodes, } \\
\text { Residual Energy, } \\
\text { Consumed Energy, } \\
\text { Network Lifetime }\end{array}$ \\
\hline
\end{tabular}

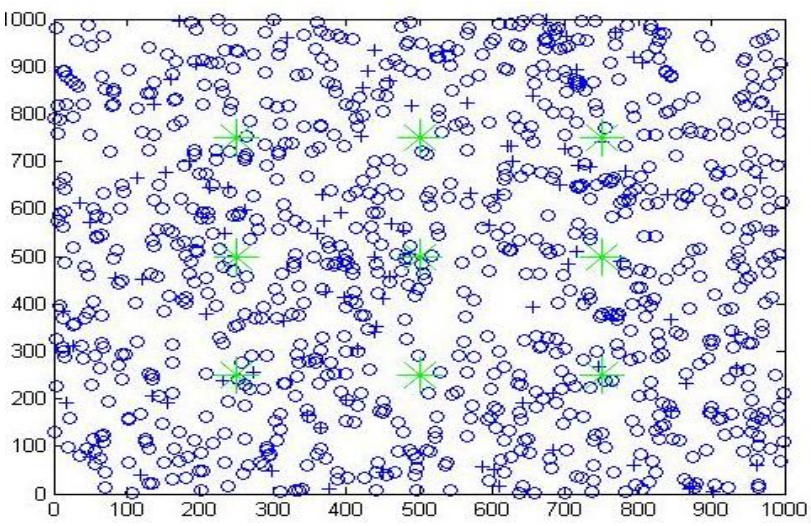

Figure. 21000 nodes network with 1000sq.m. area and 9 RNs

there is also long-distance communication due to the BS's remote position, which consumes more energy from the transmitting node as well as the routing nodes. The proposed protocol addresses these issues and consumed less energy as compared to the existing protocols. The performance analysis of residual energy and consumed energy per protocol has shown in Fig. 5 and 6. HM2LP consumed 23\% less energy as compared to the EESAA [13], EESRA[14], M-IWOCA[15], and LEACH [1]protocols and makes the network more energy efficient.

\subsection{The FND and the LND}

To estimate the impact of implementing HM2LP protocol of network stability, the FND and the last node died LND is calculated. The proposed protocol consumed less energy that's why the network runs longer as compared to the existing protocol. Fig. 7 and 8 represent the analysis for the same. It is shown that the HM2LP runs longer as compared to the existing protocols.

\subsection{The lifetime of the network}

The network lifetime period is the beginning with the first network transmission and ending after all nodes have been exhausted. The lifetime analysis of WSN is shown in Fig. 9. Experiment results show that the HM2LP live longer as compared to the rest four existing protocols. It has been found that the lifetime of the HM2LP is $12 \%$ better as compared to the EESAA [13], EESRA [14], M-IWOCA [15], and LEACH [1] protocols. 


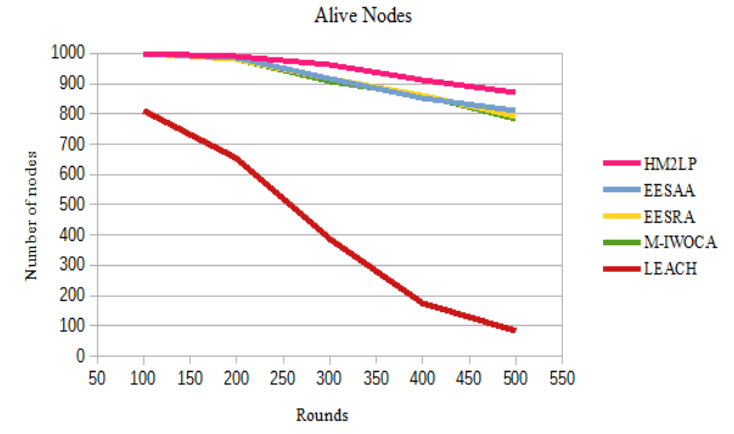

Figure. 3 Comparison of the alive node with respect to the rounds for different protocols

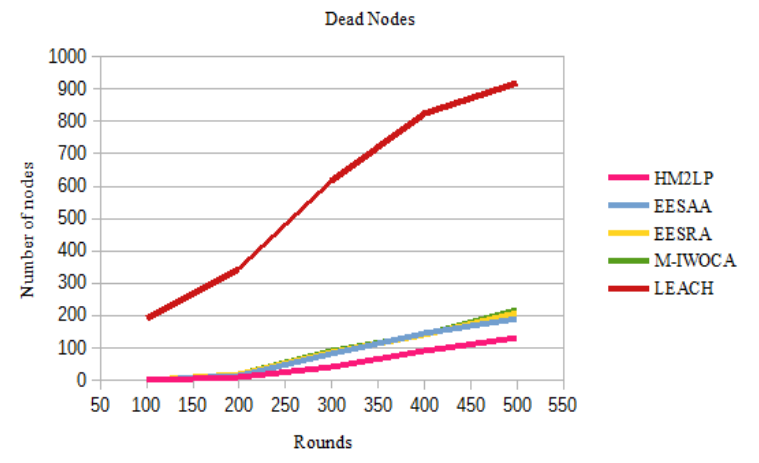

Figure. 4 Comparison of the dead node with respect to the rounds for different protocols

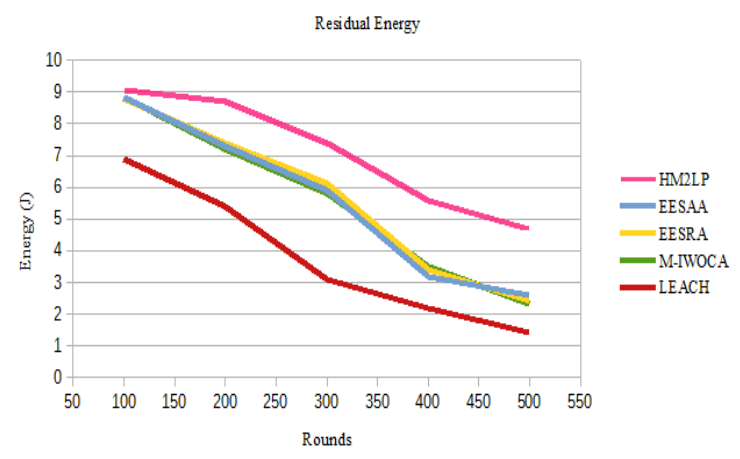

Figure. 5 Residual energy with respect to the round for different protocols

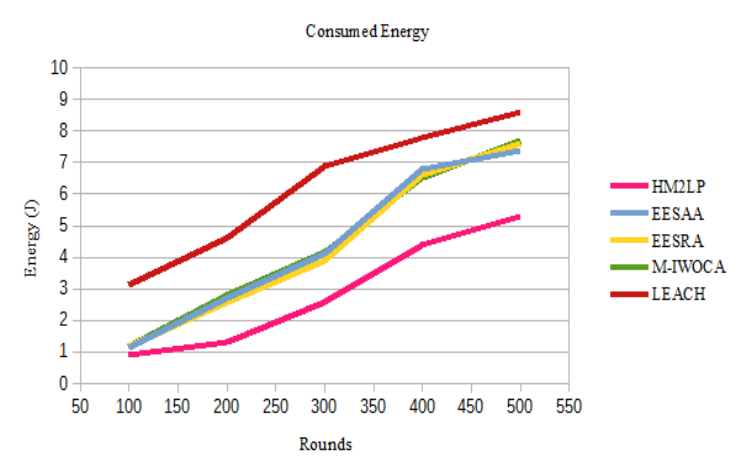

Figure. 6 Consumed energy with respect to the round for different protocols

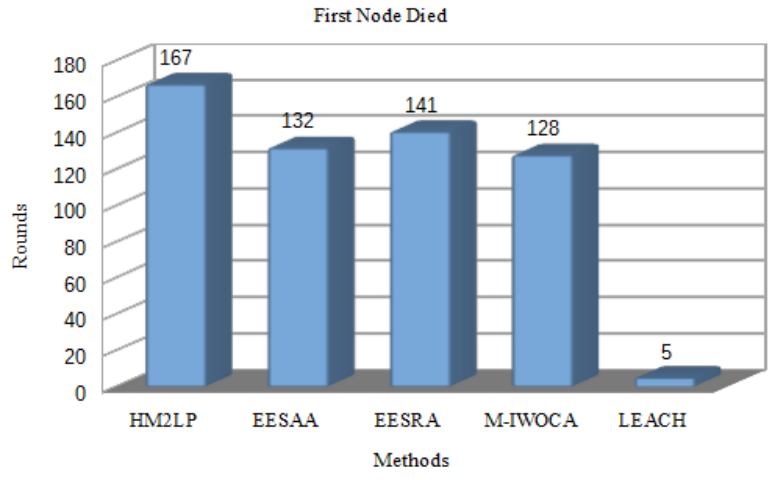

Figure. 7 FND analysis for different protocols

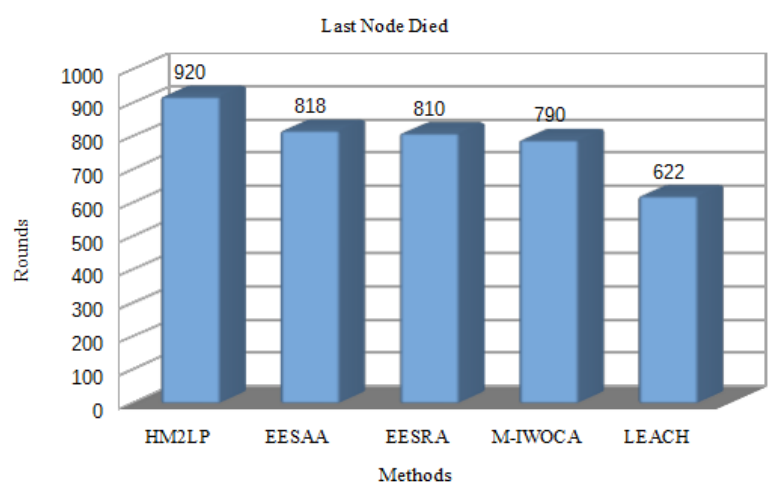

Figure. 8 LND analysis of different protocols

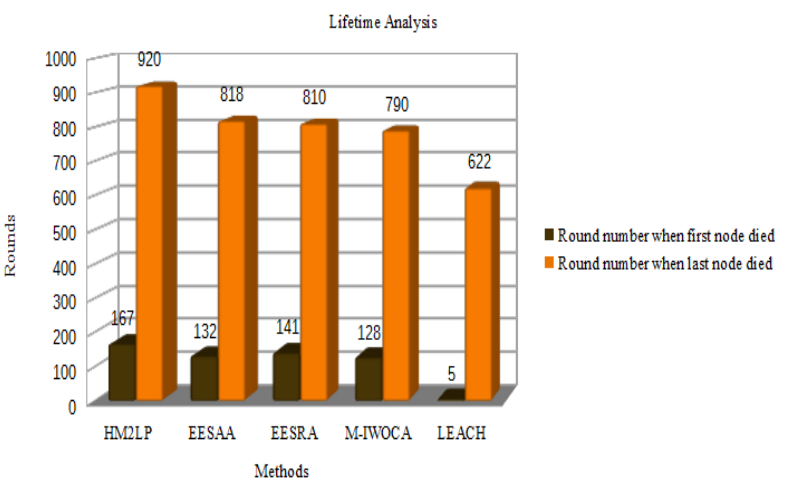

Figure. 9 WSN Lifetime of different protocols

\section{Conclusions}

The large area WSN with higher node density has more traffic due to more data generated by the large number of sensors in the network. The range of communication is required more which consumes more energy and hence less lifetime of the network. For such requirements, this paper contributes to the new HM2LP protocol. The protocol is a combination of initial clustering using K-medoid, and $\mathrm{CH}$ selection using an artificial neuro-fuzzy inference system. The $\mathrm{CH}$ selection is executed at a specific interval. This timer value is dependent on the previous load on existing $\mathrm{CH}$ due to which 
dynamicity in attempts of $\mathrm{CH}$ selection is achieved. Also, the addition of RNs in the existing WSN is responsible for the reduction in required energy in amplification due to less distance compared to BS distance. HM2LP perform superior for large area network. It consumes less energy and makes the network energy efficient. The lifetime of the HM2LP is also better. The existing protocols perform poorly for large networks, but HM2LP improves energy efficiency by approximately $12 \%$. For large networks, this article contributes to a novel HM2LP protocol that takes clustering strategy into account as well as $\mathrm{CH}$ selection is driven by timers and the usage of RN as a virtual BS. It is designed to function at the routing layer for energy efficiency, and this efficiency may be increased even further by considering bandwidth enhancement.

This may require consideration of the point coordinator function of the data link layer and especially MAC layer protocols in which bandwidth sharing and making $\mathrm{RN}$ as a virtual access point for the point coordinator function may further enhance the efficiency.

\section{Conflicts of Interest}

The authors declare no conflict of interest.

\section{Author Contributions}

Author 1: Data collection, concept, analysis, methodology, writing - original draft preparation, software, and writing - review, and editing.

Author 2: The supervision, review, investigation.

Author 3: Supervision, validation, review, investigation, and writing - review and editing.

\section{References}

[1] W. R. Heinzelman, A. Chandrakasan, and H. Balakrishnan, "Energy-Efficient Communication Protocol for Wireless Microsensor Networks", In: Proc. of 33rd IEEE International Conference on System Sciences, pp. 1-10, 2000.

[2] S. H. Kang and T. Nguyen, "Distance Based Thresholds for Cluster Head Selection in Wireless Sensor Networks", IEEE Communications Letters, Vol. 16, No. 9, pp. 1396-1399, 2012.

[3] H. V. Chaitra and G. K. Ravikumar, "Energy efficient clustering method for wireless sensor network", Indonesian Journal of Electrical Engineering and Computer Science, Vol. 14, No. 2, pp. 1039-1048, 2019.
[4] A. Rodríguez, C. D. V. Sot, and R. Velázquez, "Energy-Efficient Clustering Routing Protocol for Wireless Sensor Networks Based on Yellow Saddle Goatfish Algorithm", Mathematics, Vol. 8, No. 9, 2020.

[5] J. G. Lee, S. Chim, and H. H. Park, "EnergyEfficient Cluster-Head Selection for Wireless Sensor Networks Using Sampling-Based Spider Monkey Optimization", Sensors, Vol. 19, No. 23, pp. 1-18, 2019.

[6] A. Zahedi, "An efficient clustering method using weighting coefficients in homogeneous wireless sensor networks", Alexandria Engineering Journal, Vol. 57, No. 2, pp. 695710, 2017.

[7] A. Ranganathan and B. Rangaswamy, "Distributed Entropy Energy-Efficient Clustering algorithm for cluster head selection (DEEEC)", Journal of Intelligent \& Fuzzy Systems, Vol. 39, pp. 8139-8147, 2020.

[8] R. Pal, S. Yadav, R. Karnwal, and Aarti, "EEWC: energy-efficient weighted clustering method based on genetic algorithm for HWSNs", Complex \& Intelligent Systems, Vol. 6, pp. 391-400, 2020.

[9] B. Li and S. Park, "Maximizing the Lifetime of Wireless Sensor Networks Using Multiple Sets of Rendezvous", Mobile Information Systems, Vol. 2015, pp. 1-10, 2015.

[10] R. Pushkarna, "Rendezvous Nodes (RN Nodes) Based Cluster Head Selection and Energy Efficient Data Aggregation with Mobile Sink for Lifetime Maximization in Wireless Sensor Networks", Computing Research Repository, pp. 1-7, 2020.

[11] S. Baby and M. Soman, "Path Selection Using Rendezvous Point Method To Increase The Life Time In Wireless Sensor Networks", IOSR Journal of Electronics and Communication Engineering, pp. 48-53, 2016.

[12] A. Mehto, S. Tapaswi, and K. K. Pattanaik, "Virtual grid-based rendezvous point and sojourn location selection for energy and delay efficient data acquisition in wireless sensor networks with mobile sink", Wireless Network, Vol. 26, pp. 3763-3779, 2020.

[13] A. Ennaciri, M. Erritali, and J. Bengourram, "Load Balancing Protocol (EESAA) to improve Quality of Service in Wireless sensor network", Procedia Computer Science, Vol. 151, pp. 1140-1145, 2019.

[14] E. F. A. Elsmany, M. A. Omar, T. Wan, and A. A. Altahir, "EESRA: Energy Efficient Scalable Routing Algorithm for Wireless Sensor 
Networks", IEEE Access, Vol. 7, pp. 9697496983, 2019.

[15] R. Sharma, V. Vashisht, and U. Singh, "Fuzzy modeling based energy aware clustering in wireless sensor networks using modified invasive weed optimization", Journal of King Saud University - Computer and Information Sciences, 2019.

[16] Q. Wei, K. Bai, L. Zhou, Z. Hu, Y. Jin, and J. $\mathrm{Li}$, "A Cluster-Based Energy Optimization Algorithm in Wireless Sensor Networks with Mobile Sink", Sensors, Vol. 21, No. 3, 2021.

[17] M. Rajput, S. K. Sharma, and P. Khatri, "Improvement in LEACH Protocol for Energy Efficiency", International Journal of Innovative Technology and Exploring Engineering, Vol. 9, No. 3, pp. 2063-2069, 2020.

[18] R. G. Moghadam, M. A. Izadbakhsh, and F. S. Shabanlou, "Optimization of ANFIS network using firefly algorithm for simulating discharge coefficient of side orifices", International Journal of Applied Water Science, pp. 1-12, 2019.

[19] X. S. Yang, "Firefly Algorithms for Multimodal Optimization", Lecture Notes in Computer Science, 2009. 